This is a preprint of: "On the global asymptotic stability of difference equations satisfying a Markus-Yamabe condition", Anna Cima, Armengol Gasull, Francesc Mañosas, Publ. Mat., vol. Extra, 167-178, 2014.

DOI: [10.5565/PUBLMAT_Extra14_09]

\title{
On the global asymptotic stability of difference equations satisfying a Markus-Yamabe condition
}

\author{
Anna Cima, Armengol Gasull and Francesc Mañosas \\ Dept. de Matemàtiques, Facultat de Ciències, \\ Universitat Autònoma de Barcelona, \\ 08193 Bellaterra, Barcelona, Spain \\ cima@mat.uab.cat,gasull@mat.uab.cat,manyosas@mat.uab.cat
}

\begin{abstract}
We prove a global asymptotic stability result for maps coming from $n$-th order difference equation and satisfying a Markus-Yamabe type condition. We also show that this result is sharp.
\end{abstract}

2000 Mathematics Subject Classification: Primary: 39A11. Secondary: 37C05, 37C75.

Keywords: Global asymptotic stability, Lyapunov function, difference equation, MarkusYamabe problems.

\section{Introduction and main results}

Let $F: \mathbb{R}^{n} \longrightarrow \mathbb{R}^{n}$ be a $\mathcal{C}^{1}$ map and consider the discrete dynamical system

$$
\mathbf{x}_{k+1}=F\left(\mathbf{x}_{k}\right)
$$

LaSalle in [13] gave some possible generalizations of the sufficient conditions for global asymptotic stability (GAS) of system (1) for $n=1$. To state some of these conditions we first introduce some notations.

Let $A=\left(a_{i j}\right)$ be a $n \times n$ matrix. We denote by $\sigma(A)$ the spectrum of $A$, i.e., the set of all the eigenvalues of $A$, by $\rho(A)$ its spectral radius, $\rho(A)=\max _{\{\lambda \in \sigma(A)\}}|\lambda|$. If $A$ is real valued, then we write $|A|=\left(\left|a_{i j}\right|\right)$. Finally, we denote by $D F(\mathbf{x})=\left(D_{j}\left(F_{i}\right)(\mathbf{x})\right)$ the Jacobian matrix of $F$ at $\mathbf{x}=\left(x_{1}, x_{2}, \ldots, x_{n}\right) \in \mathbb{R}^{n}$.

The LaSalle's conditions dealing with $D F$ are:

(I) For all $\mathbf{x} \in \mathbb{R}^{n}, \rho(D F(\mathbf{x}))<1$.

(II) For all $\mathbf{x} \in \mathbb{R}^{n}, \rho(|D F(\mathbf{x})|)<1$. 
The other LaSalle's conditions are related with writing $F(\mathbf{x})=B(\mathbf{x}) \mathbf{x}$ for a $n \times n$ matrix $B(\mathbf{x})$ and then imposing some hypotheses on the eigenvalues of $B(\mathbf{x})$. They are studied in $[5,7]$ and in this work we will not consider them.

Conditions I and II are also known as Markus-Yamabe type conditions because they are similar to the condition $\sigma(D F(\mathbf{x})) \subset\{z \in \mathbb{C}: \operatorname{Re}(z)<0\}$ proposed for ordinary differential equations, see $[3,11]$ and the references therein. In [4] it is proved that condition I implies GAS for planar polynomial maps and that there are planar rational maps satisfying it having other periodic points. Later, other conditions have been added to condition I, like the one of having the infinite as a repeller, for trying to obtain GAS. Nevertheless, assuming also these additional conditions it turns out that it is possible to obtain dynamical systems for which the origin is not GAS, see [2]. In $[3,6]$ there are examples of polynomial maps defined in $\mathbb{R}^{n}, n \geq 3$, satisfying the condition and having unbounded orbits. On the other hand, in [4] it is proved that, when $F$ is polynomial, condition II implies GAS.

It is well known that for any real matrix $A, \rho(A) \leq \rho(|A|)$, see $[5,10]$. Therefore condition II is more restrictive than condition I.

In this paper we will concentrate on the problem of GAS for maps of the form

$$
F\left(x_{1}, \ldots, x_{n}\right)=\left(x_{2}, \ldots, x_{n}, f\left(x_{1}, \ldots, x_{n}\right)\right)
$$

satisfying conditions I or II. Notice that general difference equations of order $n$,

$$
x_{m+n+1}=f\left(x_{m+1}, x_{m+2}, \ldots, x_{m+n}\right),
$$

can be studied through dynamical systems generated by maps of the above form.

It is already known that even for $n=2$, condition I does not imply GAS of the fixed point for maps of the form (2). The counterexample presented in [7], based on a family introduced in [1], is given by the map

$$
F\left(x_{1}, x_{2}\right)=\left(x_{2}, 2 e^{-x_{2}^{2}}-b x_{1}\right) .
$$

For $b \in(2 / e, 1)$ it has a unique fixed point and satisfies condition I. Nevertheless, for $b \lesssim 1$ it is not GAS because, for instance, it has some periodic points of period three. In fact its behavior is very complicated because it can be seen as a perturbed twist (notice that the case $b=1$ corresponds to a twist map that preserves area). Following similar ideas, in Proposition 7 we give a simple planar rational map of the form (2), satisfying condition I for which the fixed point is not GAS.

The main contribution of this paper is the following global asymptotic stability result for difference equations satisfying condition II. Notice that the first part of the theorem also provides a more computable criterion to check whether this condition is satisfied. 
Theorem 1. Let $F: \mathbb{R}^{n} \longrightarrow \mathbb{R}^{n}$ be given by $(2)$,

$$
F\left(x_{1}, \ldots, x_{n}\right)=\left(x_{2}, \ldots, x_{n}, f\left(x_{1}, \ldots, x_{n}\right)\right)
$$

with $f: \mathbb{R}^{n} \longrightarrow \mathbb{R}$ being a $\mathcal{C}^{1}$-function. Then the following two conditions are equivalent:

$\left(H_{1}\right)$ F satisfies condition II, that is, for all $\mathbf{x} \in \mathbb{R}^{n}, \rho(|D F(\mathbf{x})|)<1$.

$\left(H_{2}\right)$ For all $\mathbf{x} \in \mathbb{R}^{n}$,

$$
\sum_{i=1}^{n}\left|D_{i}(f)(\mathbf{x})\right|<1
$$

Moreover, if they are satisfied and $F$ has a fixed point, then it is globally asymptotically stable.

The above result extends a known criterion of GAS for linear difference equations, see $[9,12,15]$. Condition (4), but only at the fixed point, already appears in [16] to obtain a result of local asymptotic stability.

Theorem 1 is sharp in the following senses:

- The hypothesis of having a fixed point can not be removed, see Lemma 5.

- If hypothesis $\left(H_{1}\right)$ is changed by $\rho(|D F(\mathbf{x})|)<K<1$, or hypothesis $\left(H_{2}\right)$ by $\sum_{i=1}^{n}\left|D_{i}(f)(\mathbf{x})\right|<K<1$, then the condition on $F$ of having a fixed point can be removed, see Theorem 6 .

- Taking the map $F$ linear, with $f\left(x_{1}, \ldots, x_{n}\right)=\sum_{i=1}^{n} a_{i} x_{i}, a_{i} \geq 0$, it is easy to see that if either $\rho(|D F(\mathbf{x})|) \geq 1$ or $\sum_{i=1}^{n}\left|D_{i}(f)(\mathbf{x})\right| \geq 1$ then the origin is no more GAS. See also [17].

The paper is structured as follows. In Section 2 we present some preliminary results about the localization of the roots of a certain class of polynomials. Section 3 is devoted to the proof of Theorem 1 and the optimality of its hypotheses. Finally in Section 4 we give a rational map of the form (2) that satisfies condition I and has a fixed point which is not GAS.

\section{Preliminary results}

The conditions that characterize whether a polynomial $P(\lambda)=\lambda^{n}+\sum_{i=0}^{n-1} a_{i} \lambda^{i}$, has all its roots inside the unit disc are also known as Jury's conditions and are extensively studied and developed, see for instance $[8,14]$ and the references therein. Next results can be seen as a simple and self-contained proof of the Jury's conditions for a particular class of polynomials. 
In fact, next lemma can also be proved applying the Perron-Frobenius Theorem([10]) to the matrix

$$
A_{P}:=\left(\begin{array}{cccccc}
0 & 1 & 0 & \cdots & 0 & 0 \\
0 & 0 & 1 & \cdots & 0 & 0 \\
\vdots & \vdots & \vdots & \ddots & \vdots & \vdots \\
0 & 0 & 0 & \cdots & 1 & 0 \\
0 & 0 & 0 & \cdots & 0 & 1 \\
-a_{0} & -a_{1} & -a_{2} & \cdots & -a_{n-2} & -a_{n-1}
\end{array}\right),
$$

because under the hypothesis of the lemma, it is non-negative and its characteristic polynomial is $(-1)^{n} P(\lambda)$. From now on we will denote by

$$
\rho(P):=\max \{|\lambda|: P(\lambda)=0\}
$$

Notice that $\rho\left(A_{P}\right)=\rho(P)$.

Lemma 2. Let $P(\lambda)=\lambda^{n}+\sum_{i=0}^{n-1} a_{i} \lambda^{i}$ be a polynomial with $a_{i} \leq 0$ for all $i$ and $\sum_{i=0}^{n-1} a_{i} \neq$ 0 . Then $P$ has a unique positive real root, $\alpha$ and $\rho(P)=\alpha$.

Proof. Applying Descartes rule, clearly $P$ has a unique positive root, say $\alpha$. Let $\beta$ be a root of $P$ and suppose to arrive to a contradiction that $|\beta|>\alpha$. Since $1=\sum_{i=0}^{n-1}\left|a_{i}\right| \beta^{i-n}$, we obtain that

$$
1 \leq \sum_{i=0}^{n-1}\left|a_{i}\right||\beta|^{i-n}<\sum_{i=0}^{n-1}\left|a_{i}\right| \alpha^{i-n}=1,
$$

which gives the desired contradiction. Therefore $\rho(P)=\alpha$.

The above lemma extends some results of [17] about the case $\sum_{i=0}^{n-1} a_{i}=1$.

Remark 3. Let $P(\lambda)=\lambda^{n}+\sum_{i=0}^{n-1} a_{i} \lambda^{i}$ be a polynomial with $a_{i} \leq 0$ for all $i$. By Lemma 2, when $\sum_{i=0}^{n-1} a_{i} \neq 0$, or directly otherwise, the following holds: given any $K>0, \rho(P)<K$ if and only if $P(K)>0$. Precisely, $P(1)>0$ is the first Jury condition which for this class of polynomials suffices to characterize whether $\rho(P)<1$.

Next result relates $\rho(P)$ and $\sum_{i=0}^{n-1}\left|a_{i}\right|$. As usual, for $a, b \in \mathbb{R}$ we denote by $\langle a, b\rangle$ the closed interval with endpoints $a$ and $b$.

Proposition 4. Let $P(\lambda)=\lambda^{n}+\sum_{i=0}^{n-1} a_{i} \lambda^{i}$ be a polynomial with $a_{i} \leq 0$ for all $i$. Then:

(i) If $\rho(P)=K<1$, then $\sum_{i=0}^{n-1}\left|a_{i}\right| \leq K$.

(ii) If $\sum_{i=0}^{n-1}\left|a_{i}\right|=K$, then $\rho(P) \in\langle K, \sqrt[n]{K}\rangle$. 
Proof. If all $a_{i}=0$, the result is trivial because this condition is equivalent to $\rho(P)=0$. So we assume that $\sum_{i=0}^{n-1} a_{i} \neq 0$.

(i) From Lemma 2 we know that $P(K)=0$, that is, $K^{n}=\sum_{i=0}^{n-1}\left|a_{i}\right| K^{i}$. Dividing this equality by $K^{n-1}$ we obtain that

$$
K=\sum_{i=0}^{n-1}\left|a_{i}\right| K^{i-n+1} \geq \sum_{i=0}^{n-1}\left|a_{i}\right|,
$$

as we wanted to show.

(ii) If $K=1$ the result follows directly. If $K<1$,

$$
\begin{aligned}
P(\sqrt[n]{K}) & =K-\sum_{i=0}^{n-1}\left|a_{i}\right| K^{\frac{i}{n}} \geq K-\sum_{i=0}^{n-1}\left|a_{i}\right|=0 \\
\frac{P(K)}{K^{n-1}} & =K-\left|a_{n-1}\right|-\sum_{i=0}^{n-2}\left|a_{i}\right| K^{i-n+1} \leq K-\left|a_{n-1}\right|-\sum_{i=0}^{n-2}\left|a_{i}\right|=0
\end{aligned}
$$

and the result is a consequence of Lemma 2 . When $K>1$ we use the same steps to obtain reversed inequalities.

\section{Results about condition II}

Proof of Theorem 1. First we will prove that $\left(H_{1}\right)$ and $\left(H_{2}\right)$ are equivalent. The characteristic polynomial of $|D F(\mathbf{x})|$ is

$$
(-1)^{n}\left(\lambda^{n}-\sum_{i=1}^{n}\left|D_{i}(f)(\mathbf{x})\right| \lambda^{i-1}\right):=(-1)^{n} P_{\mathbf{x}}(\lambda) .
$$

From Remark 3, $\rho(|D F(\mathbf{x})|)=\rho\left(P_{\mathbf{x}}\right)<1$ if and only if $P_{\mathbf{x}}(1)>0$, which is equivalent to $\sum_{i=1}^{n}\left|D_{i}(f)(\mathbf{x})\right|<1$ for all $\mathbf{x} \in \mathbb{R}^{n}$, as we wanted to see.

To prove the second part of the theorem, note that if $\mathbf{p}$ is a fixed point necessarily $\mathbf{p}=(p, \ldots, p)$ for some $p \in \mathbb{R}$. Thus conjugating $F$ by a translation we can assume that $\mathbf{p}=\mathbf{0}$.

Set $V\left(x_{1}, \ldots, x_{n}\right)=\max \left\{\left|x_{1}\right|, \ldots,\left|x_{n}\right|\right\}$. We will show that $V$ is an strict Lyapunov function for $F^{n}$ which clearly implies the desired result. For $i=1, \ldots n$ set $x_{n+i}=$ $f\left(x_{i}, \ldots, x_{n+i-1}\right)$. We claim that

$$
\left|x_{n+i}\right|<\max \left\{\left|x_{1}\right|, \ldots,\left|x_{n}\right|\right\}=V\left(x_{1}, \ldots, x_{n}\right),
$$

for all $i=1, \ldots, n$. 
For $i=1$ we have

$$
\begin{aligned}
\left|x_{n+1}\right| & =\left|\int_{0}^{1} \frac{d}{d s}\left(f\left(s x_{1}, \ldots, s x_{n}\right)\right) d s\right|=\left|\int_{0}^{1}\left(\sum_{i=1}^{n} D_{i}(f)\left(s x_{1}, \ldots, s x_{n}\right) x_{i}\right) d s\right| \\
& \leq \int_{0}^{1}\left(\sum_{i=1}^{n}\left|x_{i}\right|\left|D_{i}(f)\left(s x_{1}, \ldots, s x_{n}\right)\right|\right) d s \\
& \leq \max \left\{\left|x_{1}\right|, \ldots,\left|x_{n}\right|\right\} \int_{0}^{1}\left(\sum_{i=1}^{n}\left|D_{i}(f)\left(s x_{1}, \ldots, s x_{n}\right)\right|\right) d s<\max \left\{\left|x_{1}\right|, \ldots,\left|x_{n}\right|\right\} .
\end{aligned}
$$

Thus the claim is proved for $i=1$. Now assume that the claim is proved for $i$ and we will prove it for $i+1$. Arguing as before we obtain that

$$
\left|x_{n+i+1}\right|<\max \left\{\left|x_{i+1}\right|, \ldots,\left|x_{n+i}\right|\right\} .
$$

Therefore we have got the desired result since by the induction hypothesis we have that $\left|x_{n+j}\right|<V\left(x_{1}, \ldots, x_{n}\right)$ for all $1 \leq j \leq i$ and $\left|x_{l}\right| \leq V\left(x_{1}, \ldots, x_{n}\right)$ for $l \in\{1, \ldots, n\}$. This ends the proof of the claim.

Now, we will have

$$
V\left(F^{n}\left(x_{1}, \ldots, x_{n}\right)\right)=V\left(x_{n+1}, \ldots, x_{2 n}\right)=\max \left\{\left|x_{n+1}\right|, \ldots,\left|x_{2 n}\right|\right\}<V\left(x_{1}, \ldots, x_{n}\right),
$$

which shows that $V$ is an strict Lyapunov function for $F^{n}$. Thus $\mathbf{0}$ is GAS for $F^{n}$ which implies the same result for $F$.

Next example shows that in Theorem 1 the existence of a fixed point for $F$ cannot be deduced from the condition about $|D(F)|$.

Lemma 5. The map $F: \mathbb{R}^{n} \longrightarrow \mathbb{R}^{n}$,

$$
F\left(x_{1}, x_{2}, \ldots, x_{n}\right)=\left(x_{2}, x_{3}, \ldots, \ln \left(1+e^{\frac{x_{1}+x_{2}+\cdots+x_{n}}{n}}\right)\right),
$$

has no fixed point. Moreover, for all $\mathbf{x} \in \mathbb{R}^{n}$, the matrices $D(F)(\mathbf{x})$ and $|D(F)(\mathbf{x})|$ have all their eigenvalues with modulus less than one.

Proof. The equation $F(\mathbf{x})=\mathbf{x}$ implies that $\mathbf{x}=(x, x, \ldots, x)$ and $x=\ln \left(1+e^{x}\right)$ which has no solution. So $F$ has no fixed point.

Notice that $A(\mathbf{x}):=D(F)(\mathbf{x})=|D(F)(\mathbf{x})|$. The characteristic polynomial of $A(x)$ is

$$
P_{\mathbf{x}}(\lambda)=\lambda^{n}-\frac{1}{n} \sum_{i=1}^{n} \frac{e^{\frac{x_{1}+x_{2}+\cdots+x_{n}}{n}}}{1+e^{\frac{x_{1}+x_{2}+\cdots+x_{n}}{n}}} \lambda^{i-1} .
$$

Then $P_{\mathbf{x}}(1)>0$ and all the hypotheses of Lemma 2 hold. Therefore, for all $\mathbf{x} \in \mathbb{R}^{n}$, $\rho(A(\mathbf{x}))<1$, as we wanted to prove. 
In contrast with the above result a slightly more restrictive condition than hypothesis $\left(H_{1}\right)$, or hypothesis $\left(H_{2}\right)$, assumed in Theorem 1 forces the existence of a fixed point.

Theorem 6. Let $F: \mathbb{R}^{n} \longrightarrow \mathbb{R}^{n}$ be as in Theorem 1. Then next two conditions are equivalent:

(i) There exits $\widetilde{K}<1$ such that for all $\mathbf{x} \in \mathbb{R}^{n}, \rho(|D F(\mathbf{x})|)<\widetilde{K}$.

(ii) There exits $K<1$ such that for all $\mathbf{x} \in \mathbb{R}^{n}, \sum_{i=1}^{n}\left|D_{i}(f)(\mathbf{x})\right|<K$.

Moreover, if they are satisfied then $F$ has a fixed point which is GAS.

Proof. $(i) \Rightarrow($ ii $)$ By hypothesis,

$$
\rho(|D F(\mathbf{x})|)=\rho\left(\lambda^{n}-\sum_{i=1}^{n}\left|D_{i}(f)(\mathbf{x})\right| \lambda^{i-1}\right)<\widetilde{K}<1 .
$$

Using Proposition 4.(i) we obtain

$$
\sum_{i=1}^{n}\left|D_{i}(f)(\mathbf{x})\right|<\widetilde{K}<1
$$

(ii) $\Rightarrow\left(\right.$ i) Assume that for all $\mathbf{x} \in \mathbb{R}^{n}, \sum_{i=1}^{n}\left|D_{i}(f)(\mathbf{x})\right|<K<1$. From Proposition 4.(ii) we have that $\rho(|D F(\mathbf{x})|)<\sqrt[n]{K}<1$ and the implication follows.

Finally, in view of Theorem 1, to prove the theorem it suffices to show that under these hypotheses the map $F$ has a fixed point. Assume that (ii) holds. We need to prove that the equation $g(x)=f(x, \ldots, x)=x$ has some solution. Since

$$
\left|g^{\prime}(x)\right|=\left|\sum_{i=1}^{n} D_{i}(f)(x, \ldots, x)\right| \leq \sum_{i=1}^{n}\left|D_{i}(f)(x, \ldots, x)\right|<K<1,
$$

it follows that $g$ is a contraction and has a fixed point $p$. Then $(p, \ldots, p)$ is a fixed point for $F$. Therefore the theorem holds.

It is worth to comment that the planar maps having a fixed point not GAS and satisfying condition I given in (3) and also the one given in the forthcoming Section 4 satisfy the stronger condition $\rho(D F(\mathbf{x}))<K<1$.

\section{Results about condition I}

Following the ideas of $[1,7]$ and starting from the map (3), in this section we will construct a rational difference equation satisfying condition I and having a fixed point that is not GAS. 
Consider the family of maps

$$
F\left(x_{1}, x_{2}\right)=\left(x_{2}, g\left(x_{2}\right)-b x_{1}\right),
$$

with $g$ an smooth function satisfying that $\left|g^{\prime}(x)\right| \leq M$ for all $x \in \mathbb{R}$. It is easy to see if $M^{2}-4 b<0$ then both eigenvalues of $D F(\mathbf{x})$ are complex conjugated and $\left.\rho(D F(\mathbf{x}))\right)=b$. Therefore all maps of the above form with $b \in\left(M^{2} / 4,1\right)$ satisfy condition I.

For the map given in (3), $g(x)=2 e^{-x^{2}}$. For $b \in(2 / e, 1)$, the corresponding $F$ satisfies all the above conditions and it is seen in [7] that $F$ has a fixed point and a point of period three. So the fixed point is not GAS.

Based on the shape of this map we consider the new family of maps

$$
F\left(x_{1}, x_{2}\right)=\left(x_{2}, \frac{a}{\left(1+x_{2}^{2}\right)^{2}}-b x_{1}\right) .
$$

It is easy to see that for all $b \neq-1$ it has a unique fixed point. Since

$$
\max _{x \in \mathbb{R}} \frac{d}{d x}\left(\frac{a}{\left(1+x^{2}\right)^{2}}\right)=\sqrt{\frac{3125}{2916}}|a|,
$$

the inequalities that force that $F$ satisfies condition I read as

$$
|a|<\sqrt{\frac{11664}{3125}} \simeq 1.93 \text { and } b \in\left(\frac{3125}{11664} a^{2}, 1\right) .
$$

After a first numerical study we have obtained that for $a=9 / 5$ and $b=9 / 10$ the map (5) has a fixed point at $\mathbf{p} \simeq(0.554338,0.554338)$ and an orbit of period three at $\mathbf{q}=(-0.097039,0.241179)$. Moreover for these values of $a$ and $b$ the inequalities (6) are satisfied.

Nevertheless to prove the existence of $\mathbf{q}$ is rather complicated because we have to deal with two polynomial equations in $x$ of $y$ of degrees 21 and 89. Instead, based on these computations we change the strategy. We will force the map to have an orbit of period three at the point $\left(-\frac{1}{10}, \frac{1}{4}\right)$, which is near the point $\mathbf{q}$, and then the unknowns are $a$ and $b$. The conditions write as

$$
F^{3}\left(-\frac{1}{10}, \frac{1}{4}\right)-\left(-\frac{1}{10}, \frac{1}{4}\right)=(0,0) .
$$

We call $g(a, b)=0$ and $h(a, b)=0$ the numerators of the first and second component of the above equation. The functions $g$ and $h$ are polynomials of degrees 5 and 22, respectively. To find some values $(a, b)$ satisfying both equations we consider the new polynomial equations

$$
U(a):=\operatorname{Res}(g, h ; b)=0, \quad V(b):=\operatorname{Res}(g, h ; a)=0,
$$


where $\operatorname{Res}(\cdot, \cdot ; c)$ denotes the resultant with respect to $c$. In fact,

$$
U(a)=a^{16} U_{5}(a) \quad \text { and } \quad V(b)=\left(b^{2}+100\right)^{8} V_{5}(b),
$$

where $U_{5}$ and $V_{5}$ are given polynomials of degree 5 with huge rational coefficients. By Bolzano's Theorem it is easy to see that there are two values $a=a^{*}$ and $b=b^{*}$ satisfying

$$
U_{5}\left(a^{*}\right)=0, V_{5}\left(b^{*}\right)=0, \quad \text { and } \quad a^{*} \in\left(\frac{17}{10}, \frac{18}{10}\right), b^{*} \in\left(\frac{89}{100}, \frac{90}{100}\right) .
$$

In fact $a^{*} \simeq 1.783274$ and $b^{*} \simeq 0.897416$. Since both components of the point $(-1 / 10,1 / 4)$ are different, it is not a fixed point. Finally, notice that $a^{*}<1.8, b^{*}<1$ and

$$
b^{*}>\frac{89}{100}>\frac{3125}{11664}\left(\frac{18}{10}\right)^{2}>\frac{3125}{11664}\left(a^{*}\right)^{2} .
$$

Thus, for these values of the parameters conditions (6) hold and therefore the map (5) satisfies condition I. In short, we have proved the following result:

Proposition 7. For $a^{*}$ and $b^{*}$ satisfying (7), the map

$$
F\left(x_{1}, x_{2}\right)=\left(x_{2}, \frac{a^{*}}{\left(1+x_{2}^{2}\right)^{2}}-b^{*} x_{1}\right)
$$

fulfills condition I, has a fixed point and a periodic point of period three at $(-1 / 10,1 / 4)$. In particular the fixed point is not GAS.

The above rational example is a little bit simpler that the one of the classical example due to Szlenk and given in the appendix of [4],

$$
F\left(x_{1}, x_{2}\right)=\left(-\frac{k x_{2}^{3}}{1+x_{1}^{2}+x_{2}^{2}}, \frac{k x_{1}^{3}}{1+x_{1}^{2}+x_{2}^{2}}\right), \quad k \in(1,2 / \sqrt{3}),
$$

which has a fixed point, a periodic point of period four and also satisfies condition I.

\section{An open problem}

Taking into account the results of [4] and Theorem 1 we know that when a smooth map $F: \mathbb{R}^{n} \longrightarrow \mathbb{R}^{n}$ has a fixed point and either

- $n=1$, or

- it is polynomial, or

- it is of the form (2), 
then condition II implies GAS. Therefore it is natural to study the following Markus-Yamabe type open problem:

Let $F: \mathbb{R}^{n} \longrightarrow \mathbb{R}^{n}$ be a $\mathcal{C}^{1}$-function with a fixed point and such that condition II holds. Is then the fixed point GAS for the discrete dynamical system $\mathbf{x}_{k+1}=F\left(\mathbf{x}_{k}\right)$ ?

Until now, for all other maps that we have considered, the answer has been affirmative.

\section{Acknowledgements}

The first and second authors are partially supported by a MCYT/FEDER grant number MTM2008-03437. The third author by a MCYT/FEDER grant number MTM2008-01486. All are also supported by a CIRIT grant number 2009SGR 410.

We thank Eduardo Liz for several comments on a first version of this paper.

\section{References}

[1] S. Addas-Zanata and B. Gomes, Horseshoes for a generalized Markus Yamabe example, Qual. Theory Dyn. Syst. 10 (2011) 327-332.

[2] B. Alarcón, V. Guíñez and C. Gutierrez, Planar embeddings with a globally attracting fixed point, Nonlinear Anal. 69 (2008), 140-150.

[3] A. Cima, A. van den Essen, A. Gasull, E. Hubbers, and F. Mañosas. A polynomial counterexample to the Markus-Yamabe conjecture, Adv. Math. 131 (1997), 453-457.

[4] A. Cima, A. Gasull, and F. Mañosas. The discrete Markus-Yamabe problem, Nonlinear Anal. Ser. A: Theory Methods 35 (1999), 343-354.

[5] A. Cima, A. Gasull, and F. Mañosas. A note on LaSalle's problems, Ann. Polon. Math. 76 (2001), 33-46.

[6] A. Cima, A. Gasull, and F. Mañosas. A polynomial class of Markus-Yamabe counterexamples, Publ. Mat. 41 (1997), 85-100.

[7] A. Cima, A. Gasull, and F. Mañosas. Examples and counterexamples for MarkusYamabe and LaSalle global asymptotic stability problems, Proceedings of the Workshop Future Directions in Difference Equations. "Servizo de Publicacións da Universidade de Vigo", Col. Congresos 69, 2011, 89-96

[8] Y. Choo. An elementary proof of the Jury test for real polynomials, Automatica 47 (2011), 249-252. 
[9] C. W. Clark. A delayed-recruitment model of population dynamics, with an application to baleen whale populations, J. Math. Biol. 3 (1976), 381-391.

[10] F. R. Gantmacher, The theory of matrices. Vol. 2., Chelsea Publishing Co., New York 1959.

[11] C. Gutiérrez. A solution to the bidimensional global asymptotic stability conjecture, Ann. Inst. H. Poincaré Anal. Non Linéaire 12 (1995), 627-671.

[12] V.L. Kocić and G. Ladas, Global behavior of nonlinear difference equations of higher order with applications. Mathematics and its Applications, 256. Kluwer Academic Publishers Group, Dordrecht, 1993.

[13] J.P. LaSalle. The stability of dynamical systems. Society for Industrial and Applied Mathematics, Philadelphia, 1976.

[14] E. R. Lewis. Network models in population biology. Biomathematics, 7. SpringerVerlag, Berlin-New York, 1977.

[15] E. Liz. On explicit conditions for the asymptotic stability of linear higher order difference equations, J. Math. Anal. Appl. 303 (2005), 492-498.

[16] H. Sedaghat. Geometric stability conditions for higher order difference equations, J. Math. Anal. Appl. 224 (1998), 255-272.

[17] S. Stevic'. A note on the recursive sequence $x_{n+1}=p_{k} x_{n}+p_{k-1} x_{n-1}+\cdots+p_{1} x_{n-k+1}$, Ukraïn. Mat. Zh. 55 (2003), 570-574; translation in Ukrainian Math. J. 55 (2003), 691-697. 